\title{
SIRT1 Suppresses Doxorubicin-Induced Cardiotoxicity by Regulating the Oxidative Stress and p38MAPK Pathways
}

\author{
Yang Ruan ${ }^{a} \quad$ Chunlin Dong ${ }^{b} \quad$ Jigar Patel $^{c}$ Chao Duan ${ }^{d}$ Xinyue Wange Xi Wu \\ Yuan Cao ${ }^{e}$ Lianmei Pua Dan Lu ${ }^{a}$ Tao Shene Jian Lie \\ aBeijing Anzhen Hospital, Capital Medical University and Beijing Institute of Heart, Lung and Blood \\ Vessel Diseases, Beijing, China, 'The First People's Hospital of Jinzhou Dalian, Liaoning, China, 'Veterans \\ Affairs Medical Center and University of California, San Diego, California, Cardiology/111A, San Diego, \\ CA, USA, 'Beijing Key Laboratory of Plant Resources Research and Development, Beijing Technology \\ and Business University, Beijing, China, eKey Laboratory of Geriatrics, and Beijing Hospital and Beijing \\ Institute of Geriatrics, Ministry of Health of China, Beijing, China
}

\section{Key Words}

Sirtuin $1 \cdot$ Doxorubicin $•$ Cardiomyocyte $・$ Apoptosis $\bullet$ Oxidative stress $\bullet$ p38MAPK

\begin{abstract}
Background: SIRT1, which belongs to the Sirtuin family of NAD-dependent enzymes, plays diverse roles in aging, metabolism, and disease biology. It could regulate cell survival and has been shown to be a protective factor in heart function. Hence, we verified the mechanism by which SIRT1 regulates doxorubicin induced cardiomyocyte injury in vivo and in vitro. Methods: We analyzed SIRT1 expression in doxorubicin-induced neonatal rat cardiomyocyte injury model and adult mouse heart failure model. SIRT1 was over-expressed in cultured neonatal rat cardiomyocyte by adenovirus mediated gene transfer. SIRT1 agonist resveratrol was used to treat the doxorubicin-induced heart failure mouse model. Echocardiography, reactive oxygen species (ROS) production, TUNEL, qRT-PCR, and Western blotting were performed to analyze cell survival, oxidative stress, and inflammatory signal pathways in cardiomyocytes. Results: SIRT1 expression was down-regulated in doxorubicin induced cardiomocyte injury, accompanied by elevated oxidative stress and cell apoptosis. SIRT1 over-expression reduced doxorubicin induced cardiomyocyte apoptosis with the attenuated ROS production. SIRT1 also reduced cell apoptosis by inhibition of p38MAPK phosphorylation and caspase-3 activation. The SIRT1 agonist resveratrol was able to prevent doxorubicin-induced heart function loss. Moreover, the SIRT1 inhibitor niacinamide could reverse SIRT1's protective effect in cultured neonatal rat cardiomyocytes. Conclusions: These results support the role of SIRT1 as an important regulator of cardiomyocyte apoptosis during doxorubicin-induced heart injury, which may represent a potential therapeutic target for doxorubicin-induced cardiomyopathy.
\end{abstract}

Copyright $\odot 2015$ S. Karger AG, Basel

Tao Shen,

and Jian Li

KARGER 125
Beijing Hospital, Key Laboratory of Geriatrics,

Room 11-2 of 11th floor of Physical Examination Building, No. 1 Dahua Road, Dongdan, Dongcheng District, 100730, Beijing, (People's Republic of China)

E-Mail pkushentao@aliyun.com,E-Mail lijian@bjhmoh.cn 


\section{Introduction}

Doxorubicin, a potent chemotherapeutic agent used for a wide variety of malignancies, is associated with both acute and chronic cardiotoxicity. Since the 1970s, it has been know that doxorubicin is associated with the development of chronic systolic heart failure, which is related to the dosage and duration of treatment [1-4]. The responsible mechanisms include oxidative stress, endothelial cell injury, myofibrillar deterioration, apoptosis, and intracellular calcium dysregulation [5-8].

SIRT1 is a conserved protein, and a NAD+- dependent deacylase. Its function is linked to cellular metabolism, through its ability to sense changes of energy in the nucleus, cytoplasm, and mitochondrion [9]. SIRT1 influences the metabolism of many target proteins in various tissues, including heart, liver, muscle, adipose tissue, and endothelium $[9,10]$.

In stressed cardiomyocytes, such as oxidative stress or ischemia reperfusion, the activation of p38MAPK promotes cardiomoycyte apoptosis, which contributes to deteriorating cardiac contractile function and left ventricular remodeling during heart failure. Strong evidence has suggested that p38MAPK inhibition could attenuate cellular inflammatory reactions and protect cell from apoptosis [11-13].

In this study, we investigated the protective effects of SIRT1 on doxorubicin-induced cardiotoxicity in cultured primary neonatal rat ventricular myocytes and in adult C57BL/6J mice and elucidated the underlying mechanisms of the protective effects of SIRT1. Our results demonstrate that SIRT1 and SIRT1 agonist could suppress oxidative stress and apoptosis and ameliorate doxorubicin-induced cardiotoxicity by regulating p38MAPK pathway. These findings provide insight into the mechanisms by which SIRT1 acts as a potential therapeutic prescription for lessening the cardiotoxicity caused by doxorubicin.

\section{Materials and Methods}

\section{Animal experiment}

6-8 week old male C57BL/6J mice $(n=60)$ were randomly divided into 3 groups of 20 mice each. Normal control (Con) mice were injected and orally received an equivalent volume of placebo (saline). Doxorubicin-treated mice (DOX) were injected with a single dose of doxorubicin dissolved in saline (20mg/ $\mathrm{kg}$ i.p.) and received an orally equivalent volume of saline. Doxorubicin plus resveratrol treated mice (DOX + RES) were pretreated with resveratrol (10 mg.kg body weight-1·day-1) for 3 days by gavage before the injection and administered resveratrol for 5 additional days after the injection of the same dose doxorubicin as the DOX group [13].

All of the mice in 3 groups were euthanized 5 days after the initial injection of doxorubicin. All of the animal experiments conformed to the protocols approved by the Beijing Anzhen Hospital Animal Use and Care Committee and to the Guide for Care and Use of Laboratory Animals (NIH Publication \#85-23, revised 1996).

\section{Isolation and culture of rat cardiomyocytes}

Neonatal rat ventricular myocytes (NRVMs) were isolated from 1-day-old Sprague Dawley rats via trypsin and collagenase type II combined digestion described previously [14]. The cardiomyocytes were plated at a density of $6.6 \times 104$ cells/cm2 in high glucose DMEM supplemented with $10 \%$ FBS in the presence of $0.1 \mathrm{mM}$ 5-bromo-2-deoxyuridine.

In situ detection of reactive oxygen species (ROS)

To evaluate cardiomocyte ROS production in situ, frozen, unfixed, whole heart cross-sections or cultured NRVMs were stained with $10 \mu \mathrm{M}$ DHE (Sigma) in a dark, humidified chamber at $37^{\circ} \mathrm{C}$ for $30 \mathrm{~min}$. ROS generation was indicated by red fluorescence and was visualized with fluorescence microscopy [14]. 


\section{Echocardiography}

After doxorubicin injection for 5 days, mice were lightly anesthetized with 1\%-1.5\% isoflurane in oxygen until the heart rate stabilized at 400 to 500 beats per minute. Echocardiography was performed using Vevo 770 and Vevo 2100 (VisualSonics) instruments. Fraction shortening (FS\%) was calculated with Vevo Analysis software (version 2.2.3) as described previously [15].

\section{Histology}

Histology was performed with hearts and sections as previously described [13-15]. Tissues were processed as paraffined and subsequently analyzed by H\&E staining according to the manufacturer's protocol (Sigma-Aldrich).

Terminal deoxynucleotidyl transferase-mediated dUTP nick end labeling (TUNEL) in heart cryosections and cultured cardiac myocytes

Nuclear fragmentation was detected by TUNEL staining with an apoptosis detection kit (Roche) and the nuclei were stained by $10 \mathrm{mM}$ Hoechst 33342 as previously described. Cells (500-700) in 10 randomly chosen fields from each dish were counted to determine the percentage of apoptotic nuclei $[14,15]$. Each data indicates the results from 3 independent experiments.

\section{3-(4, 5-Dimethylthiazol-2-yl)-2, 5-diphenyltetrazolium bromide (MTT) cell viability assay}

Cell viability was examined by the MTT assay according to the instructions of the manufacturer (Roche) , as described previously [14]. NRVMs (5000 cells/ well) were plated onto 24-well plates. NRVMs were pretreated with resveratrol for $1 \mathrm{~h}$ and then treated with the indicated concentrations of doxorubicin for $24 \mathrm{~h}$. All assays were performed in triplicate.

\section{Adenovirus construction}

Recombinant adenoviruses containing mouse SIRT1 cDNA (SIRT1-Ad) were prepared using the AdEasy system according to the manufacturer's protocol. The $\beta$-gal vector adenovirus (Vector-Ad) was used as the control in this study. The SIRT1-Ad infection was performed at a multiplicity of infection (M.O.I.) of 20. Vector-Ad was added to the control groups to maintain a consistent viral load [14].

\section{Western Blot}

A Western blot was used to assess the SIRT1 protein abundance using a primary antibody directed againstSIRT1 (Millipore). In a subset of the experiments, antibodies used in this study were phosphorylationp38MAPK (p-p38MAPK), p38MAPK, caspase-3 (Cell Signaling Technology) and Bax, Bcl-2, SOD-1 (Santa Cruz). ImageJ software (NIH) was used to perform densitometry analysis [14].

\section{Statistical analysis}

The data were expressed as the mean \pm SEM. Student's $t$-tests were used to compare two conditions, and a one-way ANOVA with Bonferroni correction was used for multiple comparisons. Probability values of less than 0.05 were considered significant.

\section{Results}

Doxorubicin could induce cardiomyocyte injury

To identify the cardiomyocyte injury induced by doxorubicin and the mechanism, we assessed the cell survival rate between control and doxorubicin treated cells (Fig. 1A). As shown in Fig. 1A, compared to control, doxorubicin treatment reduced cell viability in a dosedependent manner. $1 \mu \mathrm{M}$ doxorubicin treatment led to decreased cell viability by $37.4 \pm 5.4 \%$. Therefore, we used $1 \mu \mathrm{M}$ doxorubicin for the remainder of the experiments. Similarly, TUNEL assay (Fig. 1B-C) showed doxorubicin triggered cell apoptosis in a dose-dependent manner. We also found that doxorubicin could promote p38MAPK phosphorylation and caspase- 3 activation (increased cleaved or active form of caspase-3), and decrease SIRT1 and SOD-1 expression (Fig. 1D).These results suggest that doxorubicin induces cardiomyocyte injury by promoting oxidative stress, inflammation and apoptosis. 
A.

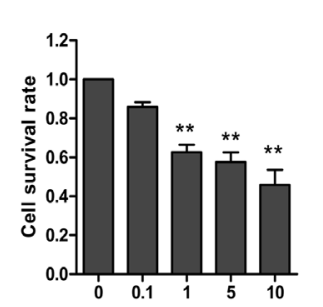

D.

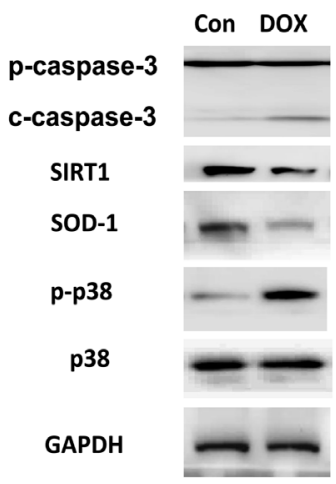

B.

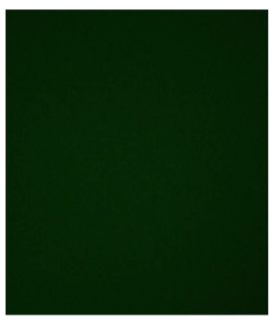

Con
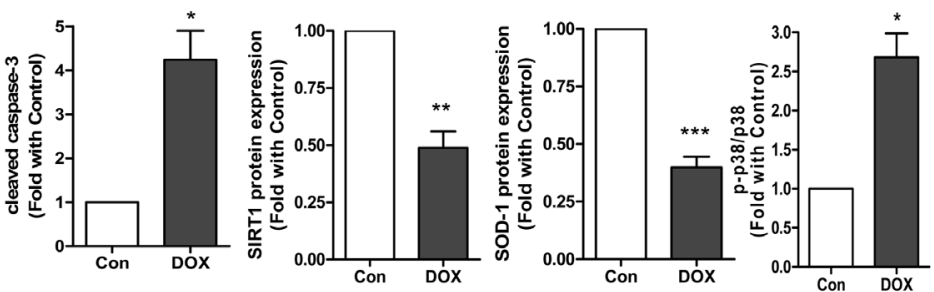

C.

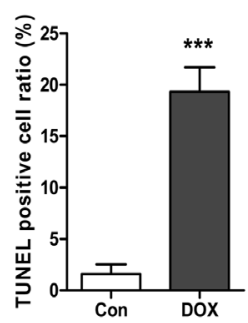

Fig. 1. Doxorubicin induces cell injury by promoting apoptosis in cardiomyocytes. A: The cell survival rate decreased with increasing concentration of doxorubicin. The NRVMs were cultured with different concentrations of doxorubicin $(0,0.1,1,5$, and $10 \mu \mathrm{M})$ for $24 \mathrm{~h}$, and the cell survival rate was demonstrated by MTT assay ( $\mathrm{n}=4)$. B-C: Representative TUNEL staining of NRVMs cultured with $1 \mu \mathrm{M}$ of doxorubicin for $24 \mathrm{~h}$ and the average data $(n=4)$. D: Pro-caspase-3(inactive form of caspase-3), cleaved caspase-3(c-caspase-3, active form of caspase-3), SIRT1, SOD-1, p-p38MAPK, p38 and GAPDH expression in control and $1 \mu \mathrm{M}$ DOX treated NRVMs for $24 \mathrm{~h}$ as assayed by Western blotting and the average data $(\mathrm{n}=4)\left({ }^{*} \mathrm{p}<0.05,{ }^{* *} \mathrm{p}<0.01\right.$, and ${ }^{* * *} \mathrm{p}$ $<0.001$ ).

Fig. 2. Over-expression of SIRT1 in NRVMs by adenovirus. NRVMs were infected with vector-Adenovirus (Vector-Ad, as the vector control) and SIRT1-Adenovirus (SIRT1-Ad) both at 20 M.O.I. for 24 h. A: SIRT1 expression in control (without adenovirus, Con), Vector-Ad and SIRT1-Ad-infected NRVMs as assayed by qRT-PCR (A) and Western blotting (B) $(\mathrm{n}=3){ }^{* *} \mathrm{p}<0.01$, and ${ }^{* * *} \mathrm{p}<$ $0.001)$.

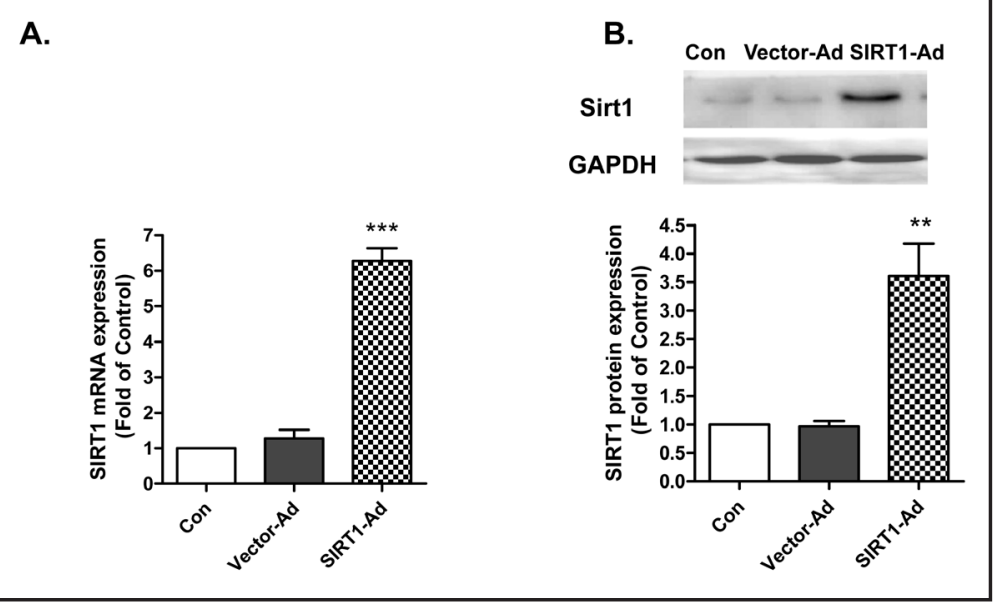

The protective effect of SIRT1 on doxorubicin-induced cardiotoxicity

For these experiments, SIRT1 was over-expressed in NRVMs using adenoviral vectors (Fig. $2 \mathrm{~A}$ and $\mathrm{B}$ ). The effectiveness of this over-expression was demonstrated by the substantially increased SIRT1 protein abundance in the SIRT1-Ad but not Vector -Ad (a vector adenovirus 


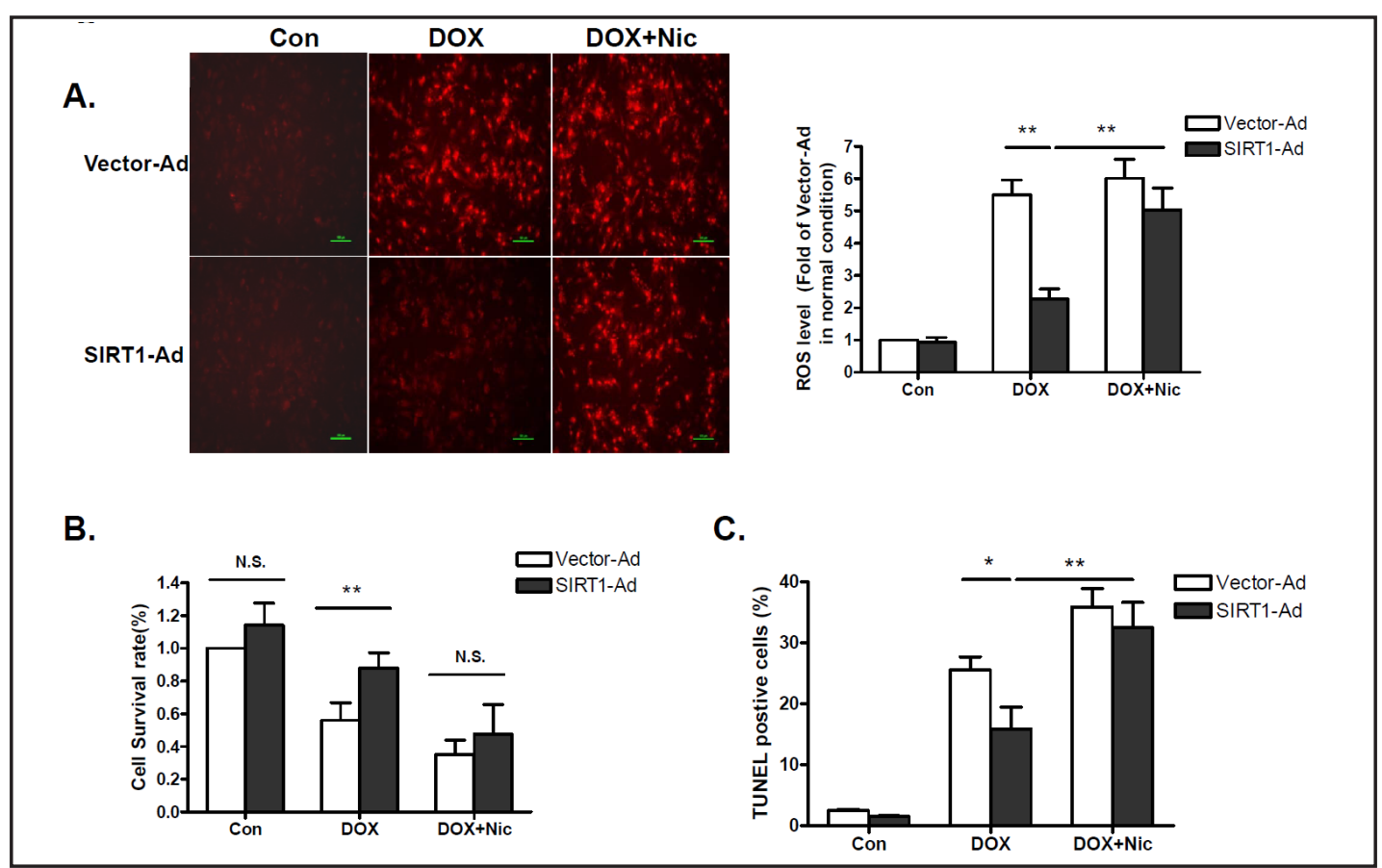

Fig. 3. SIRT1 over-expression attenuates oxidative stress-mediated NRVM apoptosis. NRVMs were infected with Vector- Ad or SIRT1-Ad (both at 20 M.O.I. for 24 h). A: Representative DHE staining of Vector-Ad and SIRT1-Ad transfected NRVMs in control (Con), $1 \mu \mathrm{M}$ doxorubicin (DOX), $1 \mu \mathrm{M}$ doxorubicin plus $40 \mathrm{mM}$ niacinamide(DOX+NIC) -treated conditions and the average data of ROS production $(\mathrm{n}=3)$. B-C: SIRT1 over-expression markedly increased cell survival and reduced cell apoptosis as assayed by the MTT assay (B) and TUNEL staining (C) , the total number of cells in each group was $800-1000$ ( $\mathrm{n}=3,{ }^{*} \mathrm{P}<0.05,{ }^{* *} \mathrm{P}<0.01$, N.S.- no significant differences).

control). This group with increased SIRT1 protein was shown to exhibit decreased ROS production, as shown in Fig. 3 ( $\mathrm{p}<0.01$ when compared with the control group). This effect on ROS production after doxorubicin was nullified by the SIRT1 antagonist niacinamide (Fig. 3A). After doxorubicin treatment, the cell survival rate was improved in the SIRT1 overexpressed group compared with adenovirus control group, whereas the group treated with $40 \mathrm{mM}$ niacinamide showed no difference between SIRT1 over-expression and control (Fig. 3B). TUNEL assay showed similar results of decreased TUNEL staining with SIRT1 overexpression unless inhibited by niacinamide (Fig. 3C). Additionally, Western blot analysis indicated that SIRT1 over-expression decreased the cleaved caspase-3 after doxorubicin treatment. Similarly, the activation of pro-inflammatory mitogen activated protein kinases (p38MAPK) was attenuated in NRVMs which had over-expressed SIRT1, when compared with the control group (Fig. 4). These experiments collectively demonstrate that SIRT1 inhibits doxorubicin-induced oxidative stress, decreases the pro-inflammation reaction, and decreases apoptosis.

\section{SIRT1 ameliorates doxorubicin-caused cardiotoxicity and apoptosis in vivo}

These experiments consisted incorporate a heart failure model using doxorubicintreated male C57BL/6 mice. As shown by H\&E staining, doxorubicin-induced heart failure was associated with decreased thickening of the left ventricular wall and increased ventricular dilatation (Fig. 5A). We quantified cardiac function by echocardiography analysis and measurement of the ejection fraction (EF). Compared with control mice, doxorubicintreated mice exhibited decreased EF, and increased level of intracellular ROS (Fig. 5B). Treatment with the SIRT1 agonist resveratrol was shown to inhibit ROS production (Fig. 


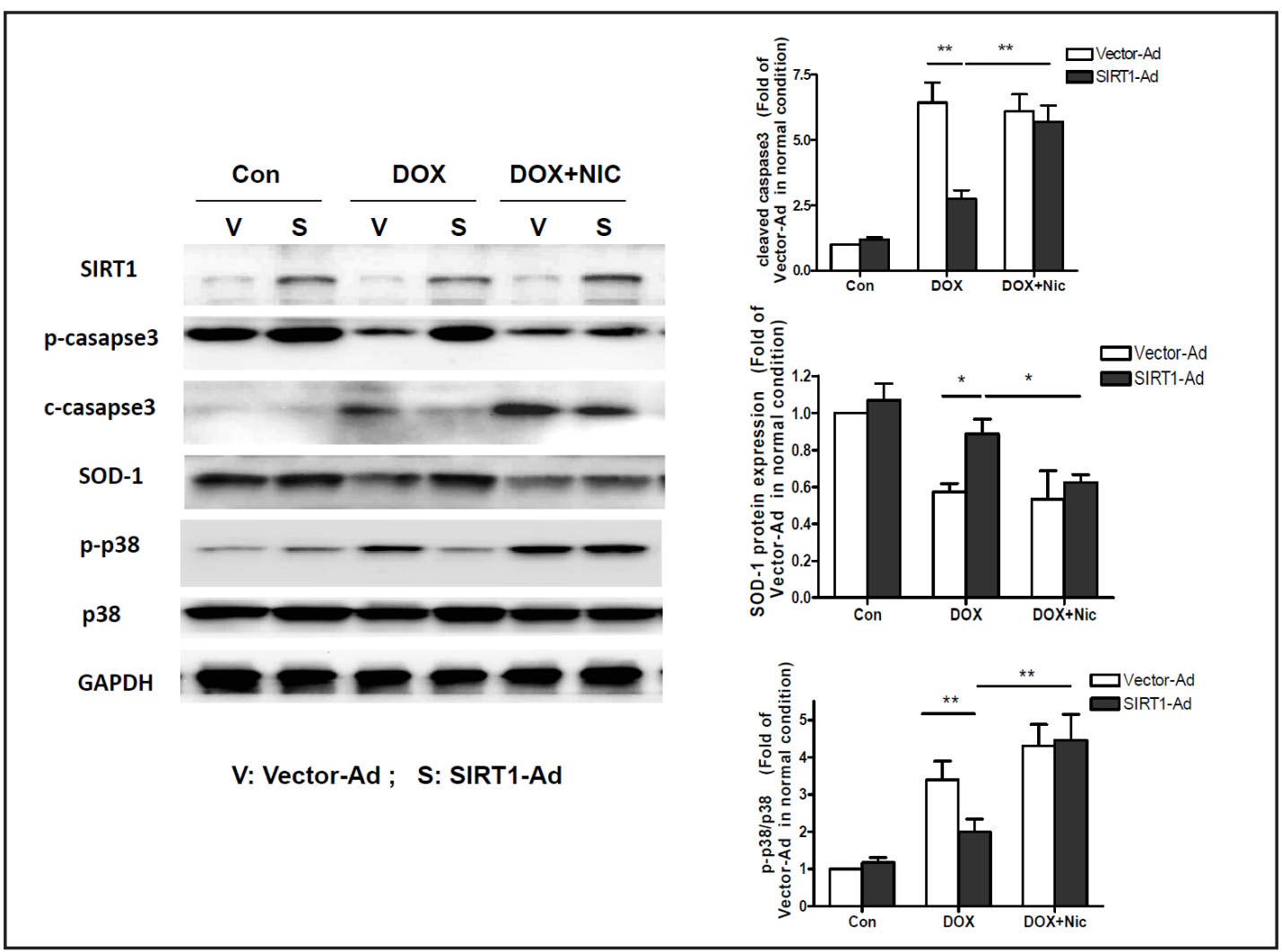

Fig. 4. SIRT1 over-expression attenuates inflammation and pro-apoptosis pathways in doxorubicin treated NRVMs. SIRT1, pro-caspase-3 (p-caspase-3), cleaved caspase3(c-capsase-3), SOD-1, p-p38, p38 and GAPDH in NRVMs which were infected with Vector-Ad(V) or SIRT1-Ad(S), and then treated with basal condition (Con), doxorubicin(DOX), or doxorubicin plus 40mM niacinamide(DOX+NIC) (n=5, *p <0.05, ** $<0.01)$.

5C). When assayed by TUNEL staining (Fig. 5D), the number of apoptotic cells was higher in the hearts of doxorubicin-treated C57BL/ 6 mice (25.04 $\pm 7.91 \%)$, compared with hearts from control mice $(1.95 \pm 1.06 \%)$. However, pretreatment with resveratrol attenuated doxorubicininduced cardiomyocyte apoptosis (Fig. 5D). Moreover, Western blot analysis revealed that SIRT1 suppressed doxorubicin-induced p38MAPK, Bax and caspase-3 activation (Fig. 6). In addition, $\mathrm{Bcl}-2$ and SOD-1 expression was dramatically upregulated in resveratrol pretreated mice (Fig. 6).

\section{Discussion}

Although doxorubicin is an extremely effective anti-neoplastic drug, the cumulative dose of doxorubicin that can be administered is often limited by cardiotoxicity. The character of Doxorubicin-induced heart failure is thinning and dilatation of the ventricular wall and a reduced ejection fraction $[16,17]$. Our results offer an explanation of the mechanism by which SIRT1 is involved in doxorubicin-induced cardiotoxicity. These experiments also demonstrate that the SIRT1 agonist resveratrol has a protective role against this toxic effect.

As an ubiquitous $\mathrm{NAD}(+)$ dependent deacetylase, SIRT1 plays an important role in physiopathology processes, metabolism, stress and aging et al. It has been showed that SIRT1 protects against neurologic disease, gastroentery disease, cancer metabolic disease and cardiovascular disease et al. [9]. But the relationship between SITRT1 and cardiotoxity is not fully clear. Our experiment found that SIRT1 agonist resveratrol administration improved cardiac function during doxorubicin induced heart injury. Our study also demonstrates that SIRT1 has a protective role in doxorubicin induced heart failure by preventing the KARGER 
Fig. 5. SIRT1 agonist resveratrol ameliorates doxorubicin-induced cardiotoxicity and apoptosis in vivo. A: Representative H\&E staining of the heart in normal mice (Con), doxorubicin treated mice (DOX), and doxorubicin plus resveratrol (DOX+RES) treated mice. $\mathrm{B}$ : The average cardiac fractional shorting (FS \%)
A.
Con
DOX
DOX+RES

assayed by Echocardiography ( $\mathrm{n}=6)$. C: The average ROS production in heart samples of normal mice (Con), doxorubicin treated (DOX), doxorubicin plus resveratrol (DOX+RES) ( $n=6)$. D: The average TUNEL staining in heart samples of normal mice (Con), doxorubicin treated (DOX), doxorubicin plus resveratrol (DOX+RES) $(\mathrm{n}=6)\left({ }^{*} \mathrm{p}<0.05,{ }^{* *} \mathrm{p}<0.01\right.$, and $\left.{ }^{* * *} \mathrm{p}<0.001\right)$.

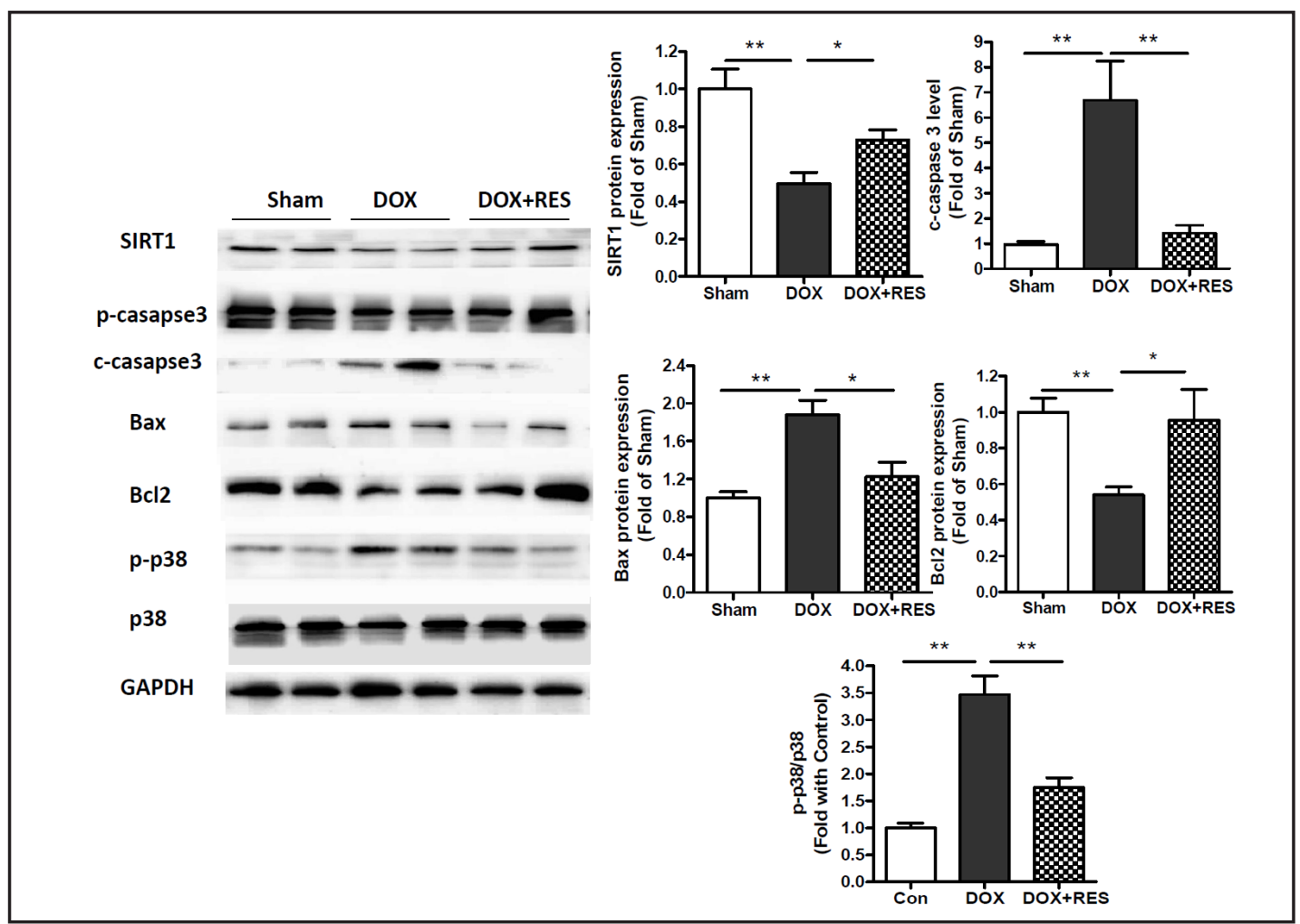

Fig. 6. SIRT1 agonist resveratrol ameliorates doxorubicin-induced cardiotoxicity and apoptosis in vivo. SIRT1, pro-caspase-3 (p-caspase-3), cleaved caspase-3(c-capsase-3), Bax, Bcl-2, p-p38MAPK, p38MAPK and GAPDH in heart samples of control mice (Con), doxorubicin treated (DOX), doxorubicin plus resveratrol (DOX+RES) $\left(\mathrm{n}=6,{ }^{*} \mathrm{P}<0.05,{ }^{* *} \mathrm{P}<0.01\right)$. 
doxorubicin-induced increase in oxidative stress and apoptosis in cardiomyocytes. To define the mechanisms for SIRT1-mediated protection in the DOX-treated cardiomyocytes, we assessed the oxidative stress and cell apoptosis in vivo and in vitro. We observed a significant decrease of ROS production in resveratrol treated mouse after doxorubicin-induced heart injury. This demonstrated that SIRT1 agonist resveratrol treatment has a protective role in doxorubicin -induced heart failure by decreasing oxidative stress.

Oxidative stress is also known to induce apoptosis by regulating inflammation signal pathway and pro-apoptotic signal pathways, which leads to ventricular dilatation both in humans and in animal models of heart failure $[18,19]$. Oxidative stress has been suggested to cause the activation of p38MAPK, which is the primary target in oxidative stress-induced heart injury [11, 20, 21]. In the present study, we have demonstrated that SIRT1 can inhibit p38MAPK phosphorylation and protect cardiomyocyte function.

Furthermore, these experiments show that the over-expression of SIRT1 by adenovirus or by the SIRT1 agonist resveratrol can inhibit the activation of caspase-3, Bax, and p38MAPK. Also, SIRT1 over-expression causes an increase in SOD-1 and Bcl-2 expression. Finally, the SIRT1 blocker niacinamide was shown to nullify these protective effects.

Collectively, these experiments show that SIRT1 plays an important role in regulating cardiac function by inhibiting oxidative stress and inflammation, and promoting cell survival, particularly in the model of doxorubicin-induced cell and function loss.

There are several limitations that require attention. First, although our study clearly indicates that SIRT1 protects cardiomyocytes from doxorubicin-induced apoptosis in cell and mouse models, it requires further investigation to determine the corresponding effect in humans. Also, resveratrol has various pharmacological effects, and SIRT1 is only one of its targets in vivo and in vitro [22,23]. Therefore, there are certainly other targets of resveratrol that require careful studies. Another limitation is the scope of the in-vivo study, which focused only on SIRT1 and oxidative stress inhibition. Further studies are required to define the role of SIRT1 in doxorubicin-induced cardiotoxicity.

\section{Conclusion}

We found that SIRT1 is involved in the protection of the heart from doxorubicin-induced oxidative stress and apoptosis, partly through the inhibition of the p38MAPK pathway. These findings are provocative and suggest SIRT1 as a potential therapeutic target to prevent cardiotoxicity caused by doxorubicin.

\section{Acknowledgements}

This work is supported by grants from National Basic Research Program of China (2012CB517502 and 2014CB910503), National Natural Science Foundation of China (81470427, 81200221 and 81270887) and Beijing Natural Science Foundation (7142142). Contributors do not warrant authorship.

\section{Disclosure Statement}

None declared.

\section{References}

1 van Dalen EC, van den BM, Caron HN, Kremer LC: Anthracycline-induced cardiotoxicity: comparison of recommendations for monitoring cardiac function during therapy in paediatric oncology trials. Eur J Cancer 2006;42:3199-3205. 
2 Dent P: The flip side of doxorubicin: Inflammatory and tumor promoting cytokines. Cancer Biol Ther 2013;14:774-775.

-3 Hershman DL, McBride RB, Eisenberger A, Tsai WY, Grann VR, Jacobson JS: Doxorubicin, cardiac risk factors, and cardiac toxicity in elderly patients with diffuse B-cell non-Hodgkin's lymphoma. J Clin Oncol 2008;26:3159-3165.

4 Longhi A, Ferrari S, Bacci G, Specchia S: Long-term follow-up of patients with doxorubicin-induced cardiac toxicity after chemotherapy for osteosarcoma. Anticancer Drugs 2007;18:737-744.

-5 Tallaj JA, Franco V, Rayburn BK, Pinderski L, Benza RL, Pamboukian S, Foley B, Bourge RC: Response of doxorubicin-induced cardiomyopathy to the current management strategy of heart failure. J Heart Lung Transplant 2005;24:2196-2201.

6 Kara G, Tasoglu 0, Dizdar O, Altundag K, Ozcakar L: Stroke due to doxorubicin-induced cardiac toxicity. Med Oncol 2009;26:506-507.

7 Lin ST, Chou HC, Chen YW, Chan HL: Redox-proteomic analysis of doxorubicin-induced altered thiol activity in cardiomyocytes. Mol Biosyst 2013;9:447-456.

8 Zhang S, Liu X, Bawa-Khalfe T, Lu LS, Lyu YL, Liu LF, Yeh ET: Identification of the molecular basis of doxorubicin-induced cardiotoxicity. Nat Med 2012;18:1639-1642.

-9 Chang HC, Guarente L: SIRT1 and other sirtuins in metabolism. Trends Endocrinol Metab 2014;25:138145.

10 Hubbard BP, Sinclair DA: Small molecule SIRT1 activators for the treatment of aging and age-related diseases. Trends Pharmacol Sci 2014;35:146-154.

11 Palomer X, varez-Guardia D, Rodriguez-Calvo R, Coll T, Laguna JC, Davidson MM, Chan TO, Feldman AM, Vazquez-Carrera M: TNF-alpha reduces PGC-1alpha expression through NF-kappaB and p38 MAPK leading to increased glucose oxidation in a human cardiac cell model. Cardiovasc Res 2009;81:703-712.

12 Martin ED, Bassi R, Marber MS: p38 MAPK in cardioprotection - are we there yet? Br J Pharmacol 2014.

13 Cao Y, Ruan Y, Shen T, Huang X, Li M, Yu W, Zhu Y, Man Y, Wang S, Li J: Astragalus Polysaccharide Suppresses Doxorubicin-Induced Cardiotoxicity by Regulating the PI3k/Akt and p38MAPK Pathways. Oxid Med Cell Longev 2014;2014:674219.

14 Shen T, Yang C, Ding L, Zhu Y, Ruan Y, Cheng H, Qin W, Huang X, Zhang H, Man Y, Liu D, Wang S, Bian Y, Xiao C, Zhao Y, Li J: Tbx20 functions as an important regulator of estrogen-mediated cardiomyocyte protection during oxidative stress. Int J Cardiol 2013;168:3704-3714.

15 Shen T, Aneas I, Sakabe N, Dirschinger RJ, Wang G, Smemo S, Westlund JM, Cheng H, Dalton N, Gu Y, Boogerd CJ, Cai CL, Peterson K, Chen J, Nobrega MA, Evans SM: Tbx20 regulates a genetic program essential to adult mouse cardiomyocyte function. J Clin Invest 2011;121:4640-4654.

16 Fatima N, Zaman MU, Hashmi A, Kamal S, Hameed A: Assessing adriamycin-induced early cardiotoxicity by estimating left ventricular ejection fraction using technetium-99m multiple-gated acquisition scan and echocardiography. Nucl Med Commun 2011;32:381-385.

17 McKillop JH, Bristow MR, Goris ML, Billingham ME, Bockemuehl K: Sensitivity and specificity of radionuclide ejection fractions in doxorubicin cardiotoxicity. Am Heart J 1983;106:1048-1056.

18 Nabeebaccus A, Zhang M, Shah AM: NADPH oxidases and cardiac remodelling. Heart Fail Rev 2011;16:512.

19 Nakamura K, Kusano KF, Matsubara H, Nakamura Y, Miura A, Nishii N, Banba K, Nagase S, Miyaji K, Morita H, Saito H, Emori T, Ohe T: Relationship between oxidative stress and systolic dysfunction in patients with hypertrophic cardiomyopathy. J Card Fail 2005;11:117-123.

20 Yeh CC, Li H, Malhotra D, Turcato S, Nicholas S, Tu R, Zhu BQ Cha J, Swigart PM, Myagmar BE, Baker AJ, Simpson PC, Mann MJ: Distinctive ERK and p38 signaling in remote and infarcted myocardium during postMI remodeling in the mouse. J Cell Biochem 2010;109:1185-1191.

21 Consoli C, Gatta L, Iellamo F, Molinari F, Rosano GM, Marlier LN: Severity of left ventricular dysfunction in heart failure patients affects the degree of serum-induced cardiomyocyte apoptosis. Importance of inflammatory response and metabolism. Int J Cardiol 2013;167:2859-2866.

22 Poulsen MM, Jorgensen JO, Jessen N, Richelsen B, Pedersen SB: Resveratrol in metabolic health: an overview of the current evidence and perspectives. Ann N Y Acad Sci 2013;1290:74-82.

$\checkmark 23$ Marchal J, Pifferi F, Aujard F: Resveratrol in mammals: effects on aging biomarkers, age-related diseases, and life span. Ann N Y Acad Sci 2013;1290:67-73. 